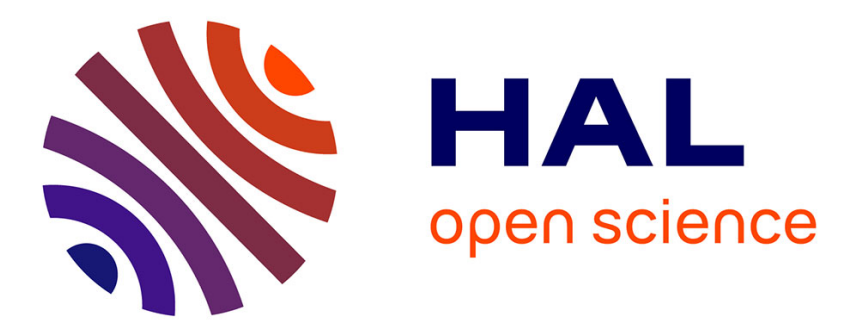

\title{
Acoustic wave propagation and internal fields in rigid frame macroscopically inhomogeneous porous media
}

Laurent de Ryck, Walter Lauriks, Zine El Abiddine Fellah, Armand Wirgin, J.-P. Groby, Philippe Leclaire, Claude Dépollier

\section{To cite this version:}

Laurent de Ryck, Walter Lauriks, Zine El Abiddine Fellah, Armand Wirgin, J.-P. Groby, et al.. Acoustic wave propagation and internal fields in rigid frame macroscopically inhomogeneous porous media. Journal of Applied Physics, 2007, 102 (2), pp.024910.1-024910.11. 10.1063/1.2752135 . hal00461020

\author{
HAL Id: hal-00461020 \\ https://hal.science/hal-00461020
}

Submitted on 12 Feb 2022

HAL is a multi-disciplinary open access archive for the deposit and dissemination of scientific research documents, whether they are published or not. The documents may come from teaching and research institutions in France or abroad, or from public or private research centers.
L'archive ouverte pluridisciplinaire HAL, est destinée au dépôt et à la diffusion de documents scientifiques de niveau recherche, publiés ou non, émanant des établissements d'enseignement et de recherche français ou étrangers, des laboratoires publics ou privés. 


\title{
Acoustic wave propagation and internal fields in rigid frame macroscopically inhomogeneous porous media
}

\author{
L. De Ryck and W. Lauriks ${ }^{\text {a) }}$ \\ Laboratorium voor Akoestiek en Thermische Fysica, K. U. Leuven, Celestijnenlaan 200D, B-3001 Heverlee, Belgium
}

Z. E. A. Fellah and A. Wirgin

Laboratoire de Mécanique et d'Acoustique, UPR 7051 CNRS, 31 Chemin Joseph Aiguier, 13009 Marseille, France

J. P. Groby

Centre de Mathématiques Appliquées, UMR 7641 CNRS/Ecole Polytechnique, 91128 Palaiseau Cedex, France

\section{P. Leclaire}

Laboratoire de Recherche en Mécanique et Acoustique, ISAT/Université de Bourgogne, 49 rue Mademoiselle Bourgeois, Boîte Postale

31-58027 Nevers Cedex, France

\section{Depollier}

Laboratoire d'Acoustique de l'Université du Maine, UMR 6613 CNRS, Avenue Olivier Messiaen, 72000 Le Mans, France

A wave propagation model in macroscopically inhomogeneous porous media is derived from the alternative Biot's theory of 1962. As a first application, the wave equation is reduced and solved in the case of rigid frame inhomogeneous porous materials. The pressure field, as well as the reflection and transmission coefficients, are obtained numerically using a wave splitting and "transmission" Green's functions approach (WS-TGF). To validate both the wave equation and the method of resolution at normal and oblique incidence, results obtained by the WS-TGF method are compared to those calculated by the classical transfer matrix method and to experimental measurements for a known two-layered porous material, considered as a single inhomogeneous layer. Discussions are then given of the reflection and transmission coefficients for various inhomogeneity profiles as well as of the internal pressure field.

\section{INTRODUCTION}

Acoustic wave propagation in porous materials was mainly studied, after Biot's theory ${ }^{1,2}$ was elaborated, in order to deal with sound absorption, material properties characterization, etc. This concerns a wide range of applications: building acoustics (concrete walls, rockwools, plastic foams), medicine (osteoporosis), ${ }^{3}$ oil prospection, and civil engineering (vibration reduction).

The study of wave propagation in macroscopically inhomogeneous porous media was initially motivated by: (i) the design of sound absorbing porous materials with optimal material and geometrical property profiles ${ }^{4}$ and (ii) the retrieval of the spatially varying material parameters of industrial foams. These, and other inverse problems, are of great importance in connection with the characterization of the mechanical properties of naturally occurring macroscopically inhomogeneous porous materials, such as bones. The literature on inhomogeneous media is extensive in several fields of physics, from optics and electromagnetism ${ }^{5,6}$ to acoustics ${ }^{7-9}$ and geophysics. ${ }^{10}$ Many natural ${ }^{11}$ and man-made materials are porous, and therefore heterogeneous at a microscopic scale. ${ }^{1,12}$

\footnotetext{
a)Electronic mail: walter.lauriks@fys.kuleuven.be
}

The first part of the present article offers some arguments and bibliographic elements concerning the most appropriate set of equations to model the propagation of acoustic waves in macroscopically inhomogeneous porous materials. Porous media are biphasic materials with a fluid phase and a solid phase, also called the skeleton or the frame. Homogeneous porous materials are well described by the first work of Biot ${ }^{1,2}$ and later contributions. ${ }^{13-15}$ At the microscopic scale of the pore, all porous materials-rockwools, polyurethane foams, concrete, sandstones-are heterogeneous. In the usual analysis of acoustic wave propagation in such materials, it is assumed that the wavelength is greater than the average size of the heterogeneity. The microscopic physical properties of the porous material are also homogenized. ${ }^{1,12}$ However, a spatial variation of the homogenized physical properties can also exist at the macroscopic scale. The equations of motion for macroscopically inhomogeneous porous materials are derived herein from the alternative formulation of Biot's theory. ${ }^{2,16}$.

As a first verification of the validity of these equations, acoustic wave propagation in rigid frame porous materials solicited by high frequency waves is considered. ${ }^{13,15-18}$ The equations of motion are approximated and the behavior of porous media is found to formally match that of an effective inhomogeneous fluid. Such an approximation is particularly 
appropriate to model wave propagation in polyurethane foams saturated by air (or by any other light fluid) even if the frequency range to validate this assumption is band limited at high frequency by the diffusion limit (when the wavelength is of the order of, or smaller than, the pore size, multiple diffusion effects appear), ${ }^{19}$ and at low frequency by the Biot characteristic frequency, which defines the limit between low and high frequency range in the Biot models.

Subsequently, a numerical procedure is proposed to solve the wave equation in a macroscopically inhomogeneous rigid frame porous medium. The wave equation in an inhomogeneous medium can be solved in a variety of manners: via the wave splitting method, ${ }^{6,16,20}$ the transfer matrix method $^{15,21}$ (for piecewise constant media), integral methods, ${ }^{22-24}$ or purely numerical (e.g., finite-element ${ }^{25}$ or finite-difference ${ }^{26}$ ) methods. The methods dedicated to inverse problems are wave splitting ${ }^{27-29}$ and linearization ${ }^{23,30,31}$ techniques deriving from the integral formalism. We will focus here on the wave splitting-transmission Green's functions approach (WS-TGF), which was employed in electromagnetic scattering theory initially in the time domain ${ }^{32}$ and then adapted to the frequency domain. ${ }^{6}$ Transmission Green's functions are an alternative approach to the classical Green's functions. ${ }^{33}$ The method is based on the factorization of the wave equation, which leads to a linearizable system of first order differential equations. The accuracy of both the wave equation and the WS-TGF method employed to solve the latter is established on the example of a known twolayered porous material, considered as a single inhomogeneous material slab with a sudden change in the properties values. Numerical results of the WS-TGF method are compared to calculations of the transfer matrix method (TMM), ${ }^{15}$ which is particularly suitable to solve problems involving a layered configuration.

Numerical results are also compared to experimental measurements at both normal and oblique incidence. The experiment consists in recording reflected and transmitted ultrasonic waves traveling through the chosen two-layered porous medium. The pressure field inside the inhomogeneous slab is also obtained by the numerical method.

Finally, practical issues in material design for engineering applications are treated, by comparing the internal, reflected, and transmitted pressure fields for macroscopically inhomogeneous porous slabs with various inhomogeneity profiles that are either continuous or discontinuous.

\section{EQUATIONS OF MOTION IN INHOMOGENEOUS POROELASTIC MATERIALS}

As pointed out by several authors, $2,16,34,35$ the generalized formulation of the Biot theory ${ }^{2}$ is suitable to macroscopically inhomogeneous porous media and also to take into account anisotropy and viscoelastic frames.

\section{A. Equations of motion}

From Biot's alternative theory, ${ }^{2}$ the constitutive strainstress relations in an initially stress-free, statistically isotropic porous material are

$$
\sigma_{i j}=2 \mu \epsilon_{i j}+\delta_{i j}\left(\lambda_{c} \theta-\alpha M \zeta\right),
$$

$$
p=M(-\alpha \theta+\zeta)
$$

where $\sigma_{i j}$ is the total stress tensor and $p$ the fluid pressure in the pores; $\delta_{i j}$ denotes the Kronecker symbol; $\theta=\nabla \cdot \mathbf{u}$ and $\zeta$ $=-\nabla \cdot \mathbf{w}$ are, respectively, the dilatation of the solid and the variation of fluid content, where $\mathbf{u}$ is the solid displacement and $\mathbf{w}=\phi(\mathbf{U}-\mathbf{u})$ the fluid/solid relative displacement ( $\mathbf{U}$ is the fluid displacement); $\phi$ is the porosity; $\epsilon_{i j}=1 / 2\left(u_{i, j}+u_{j, i}\right)$ is the strain tensor of the solid phase under the hypothesis of small deformations; $\lambda_{c}=\lambda+\alpha^{2} M$, where $\lambda, \mu$, are the Lamé coefficients of the solid phase, $M$ is an additional elastic parameter; and $\alpha$ is a coefficient of elastic coupling. These parameters were defined by Biot and Willis. ${ }^{36}$

In the absence of body forces, the conservation of momentum and the generalized Darcy's law lead to the following equations:

$$
\begin{aligned}
& \nabla \cdot \sigma=\rho \ddot{\mathbf{u}}+\rho_{f} \ddot{\mathbf{w}}, \\
& -\nabla p=\rho_{f} \ddot{\mathbf{u}}+m \ddot{\mathbf{w}}+\frac{\eta}{\kappa} F \dot{\mathbf{w}},
\end{aligned}
$$

wherein the dot and double dots refer, respectively, to first and second order time derivatives; $\rho_{f}$ is the density of the fluid in the pores, $\rho$ is the bulk density of the porous medium, such that $\rho=(1-\phi) \rho_{s}+\phi \rho_{f}$, in which $\rho_{s}$ is the density of the solid; $m=\rho_{f} \tau_{\infty} / \phi$ is a mass parameter defined by Biot, ${ }^{2}$ $\tau_{\infty}$ is the tortuosity, $\eta$ is the viscosity of the fluid, $\kappa$ is the permeability, and $F$ is the viscosity correction function. The ratio $\eta / \kappa$ is also known in engineering acoustic applications as the flow resistivity and is denoted by $R_{f}$.

It is important to note that the viscous correction function $F$ in Eq. (4) is a convolution operator in the time domain. This operator incorporates the characteristic viscous length $\Lambda$ introduced by Johnson et al. ${ }^{13}$ The elastic parameter $M$ in Eqs. (1) and (2) is also a convolution operator in the time domain and incorporates the characteristic thermal length $\Lambda^{\prime}$ defined by Champoux and Allard. ${ }^{17}$ Both characteristic lengths are geometrical parameters of the pore-size distribution inside the porous sample. The thermal characteristic length represents a measure of the average pore size (although the "pore" is not always straightforwardly defined) while the viscous characteristic length corresponds to the average size of the "constrictions" in the porous medium, ${ }^{15}$ i.e., the average distance between pore walls in the narrower areas of the pore volume.

For simplicity, spatially varying parameters will only depend on $x$ in the following. In the ealier Eqs. (1)-(4), the $x$-dependent mechanical parameters are $\lambda, \mu, \lambda_{c}, \alpha, M, \phi, \rho$, $m, \tau_{\infty}, \kappa, F, \Lambda$, and $\Lambda^{\prime}$.

\section{B. Wave equations in terms of the displacements $\mathrm{u}$ and $\mathbf{w}$}

The conservation of the momentum relations (3) and (4) involve the divergence of the total stress tensor $\boldsymbol{\sigma}=\sigma_{i j}$ and the gradient of the fluid pressure $p$. In particular, from Eq. (1), the divergence of the total stress tensor $\boldsymbol{\sigma}=\sigma_{i j}$ can be 


$$
\begin{aligned}
\nabla \cdot \boldsymbol{\sigma}= & \nabla\left[\left(\lambda_{c}+2 \mu\right) \nabla \cdot \mathbf{u}\right]-\nabla \times(\mu \nabla \times \mathbf{u}) \\
& -2(\nabla \mu)(\nabla \cdot \mathbf{u})+2[\nabla \mu \times(\nabla \times \mathbf{u})] \\
& +2(\nabla \mu \cdot \nabla) \mathbf{u}+\nabla(\alpha M \nabla \cdot \mathbf{w}) .
\end{aligned}
$$

Equations (5) and $\nabla \cdot(2)$, together with Eqs. (3) and (4), lead to the following wave equations in macroscopically inhomogeneous porous materials:

$$
\begin{aligned}
& \nabla\left[\left(\lambda_{c}+2 \mu\right) \nabla \cdot \mathbf{u}+\alpha M \nabla \cdot \mathbf{w}\right]-\nabla \times[\mu \nabla \times \mathbf{u}] \\
& -2 \nabla \mu \nabla \cdot \mathbf{u}+2 \nabla \mu \times(\nabla \times \mathbf{u})+2(\nabla \mu \cdot \nabla) \mathbf{u} \\
& =\rho \ddot{\mathbf{u}}+\rho_{f} \ddot{\mathbf{w}}, \\
& \nabla(M \nabla \cdot \mathbf{w}+\alpha M \nabla \cdot \mathbf{u})=\rho_{f} \ddot{\mathbf{u}}+m \ddot{\mathbf{w}}+\frac{\eta}{\kappa} F \dot{\mathbf{w}},
\end{aligned}
$$

wherein the $x$ dependence of the constitutive parameters has been removed to simplify the notations.

When the fluid phase vanishes, the porosity is $\phi=0$ and the elastic coupling term is $\alpha=0$. This implies that $\mathbf{w}=0$, $\lambda_{c}=\lambda$, and $\rho=\rho_{s}$. The second equation of the system (6) vanishes and the first equation reduces to the equation of motion of an inhomogeneous elastic solid as given in Robins ${ }^{9}$

$$
\begin{gathered}
\nabla[(\lambda+2 \mu) \nabla \cdot \mathbf{u}]-\nabla \times(\mu \nabla \times \mathbf{u})-2(\nabla \mu)(\nabla \cdot \mathbf{u}) \\
+2[\nabla \mu \times(\nabla \times \mathbf{u})]+2(\nabla \mu \cdot \nabla) \mathbf{u}=\rho_{s} \ddot{\mathbf{u}} .
\end{gathered}
$$

When the solid phase vanishes, the porosity is $\phi=1$ and the tortuosity is $\tau_{\infty}=1$. This implies that $\mathbf{w}=\mathbf{U}-\mathbf{u}, m=\rho_{f}, \rho$ $=\rho_{f}$, the Lamé coefficients $\lambda$ and $\mu$ vanish, and the elastic coupling term is $\alpha=1 .^{36}$ Moreover, the viscous correction function $F$ vanishes, so that both equations in Eq. (6) reduce to the equation $\nabla(M \nabla \cdot \mathbf{U})=\rho_{f} \ddot{\mathbf{U}}$ in which are included the Euler equation and stress-strain relation in an inhomogeneous fluid. With the help of Eq. (2), these relations are

$$
\begin{aligned}
& -\nabla p=\rho_{f} \ddot{\mathbf{U}}, \\
& -p=M \nabla \cdot \mathbf{U},
\end{aligned}
$$

wherein $M=K(x, t)$ is the inhomogeneous bulk modulus of the fluid and $\rho_{f}(x)$ the $x$-dependent density.

\section{Wave equation under the rigid frame assumption in terms of the pressure field}

In the rigid frame approximation, the displacement of the skeleton, i.e., the solid phase, is $\mathbf{u} \simeq 0$. The usual unknown of the wave equation when the assumption of a rigid frame is valid is the pressure field $p . p$ does not appear in Eqs. (1) and (3), while it appears in both Eqs. (2) and (4). The latter can be rewritten in the frequency domain, and the behavior of the porous medium is found to formally match that of an equivalent fluid

$$
\begin{aligned}
& -\nabla p=j \omega \rho_{e}(x, \omega) \phi(x) \dot{\mathbf{U}}, \\
& -j \omega p=K_{e}(x, \omega) \nabla .[\phi(x) \dot{\mathbf{U}}],
\end{aligned}
$$

where $\quad \rho_{e}(x, \omega)=m(x)-j(\eta / \kappa \omega) F(x, \omega) \quad$ and $\quad K_{e}(x, \omega)$ $=M(x, \omega)$ are, respectively, the effective density and bulk modulus of the inhomogeneous equivalent fluid. The unknowns reduce to the pressure field $p$ and the fluid flow $\phi \dot{\mathbf{U}}$. The latter are consistent with the boundary conditions between two porous materials, ${ }^{37}$ i.e., continuity of the fluid pressure and of the component of the fluid flow normal to the boundary.

According to the definition of $m$ and $M,{ }^{15,38}$ the effective density and bulk modulus are

$$
\begin{aligned}
& \rho_{e}(x, \omega)=\rho_{f} \frac{\tau_{\infty}(x)}{\phi(x)}\left[1-j \frac{R_{f}(x) \phi(x)}{\rho_{f} \tau_{\infty}(x) \omega} F(x, \omega)\right], \\
& K_{e}(x, \omega)=\frac{\gamma P_{0} / \phi(x)}{\gamma-(\gamma-1)\left[1-j \frac{R_{f}(x) \phi(x)}{\rho_{f} \tau_{\infty}(x) B^{2} \omega} G\left(x, B^{2} \omega\right)\right]^{-1}},
\end{aligned}
$$

wherein $\gamma$ is the specific heat ratio, $P_{0}$ is the atmospheric pressure, and $B^{2}=P_{r}$ is the Prandtl number. $F(x, \omega)$ and $G\left(x, B^{2} \omega\right)$ are the well-defined correction functions of the Johnson-Allard rigid frame model $^{13,15}$

$$
\begin{aligned}
& F(x, \omega)=\sqrt{1+j 4 \frac{\eta \rho_{f} \tau_{\infty}^{2}(x)}{R_{f}(x)^{2} \phi(x)^{2} \Lambda^{2}(x)} \omega,} \\
& G\left(x, B^{2} \omega\right)=\sqrt{1+j 4 \frac{\eta \rho_{f} \tau_{\infty}^{2}(x)}{R_{f}(x)^{2} \phi(x)^{2} \Lambda^{\prime 2}(x)} B^{2} \omega .}
\end{aligned}
$$

The relevant parameters describing the propagation of acoustic waves in macroscopically inhomogeneous rigid frame porous materials are the porosity $\phi(x)$, the tortuosity $\tau_{\infty}(x)$, the flow resistivity $R_{f}(x)=\eta / \kappa(x)$, the characteristic viscous length $\Lambda(x)$, and the characteristic thermal length $\Lambda^{\prime}(x)$.

Finally, combining Eqs. (10) and (11), the Helmholtz equation in terms of $p$ is obtained

$$
\nabla \cdot\left[\frac{1}{\rho_{e}(x, \omega)} \nabla p\right]+\frac{\omega^{2}}{K_{e}(x, \omega)} p=0 .
$$

This is an inhomogeneous fluid-like Helmholtz equation, which can be solved in a variety of manners. ${ }^{22-25,31}$ In the following, we present the "vacuum" WS-TGF in the frequency domain.

\section{NUMERICAL CALCULATION OF THE PRESSURE FIELD}

The wave splitting method was mainly used to solve scattering problems in several fields of physics, especially in electromagnetism, initially in the time domain ${ }^{27-29,32,33}$ and then adapted to the frequency domain. ${ }^{6}$ Maxwell's equations are similar to the equations of motion in fluid-like materials and to Eqs. (10) and (11) in rigid frame porous materials. In order to avoid convolution products in terms of time and fractional derivatives, ${ }^{39}$ the direct scattering problem is treated in the frequency domain. Also, rather than solve directly for $p(x, t)$ we will focus on its Fourier transform $p(x, \omega)$ defined by

$$
p(x, t)=\int_{-\infty}^{\infty} p(x, \omega) \exp (j \omega t) d \omega .
$$




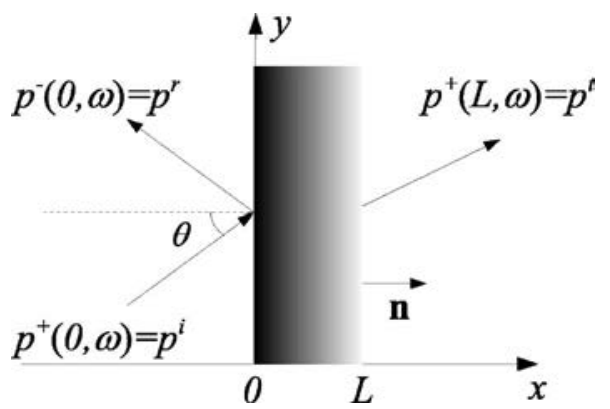

FIG. 1. Slab of macroscopically inhomogeneous porous material.

The wave splitting technique, together with the invariant imbedding method and Green's function approach, leads to the direct scattering solution by calculating the internal pressure field. Then, the reflected and transmitted fields are numerically obtained.

In the following sections, a macroscopically onedimensional inhomogeneous porous slab of thickness $L$, solicited by an incident plane wave propagating initially in the air medium (ambient medium), is considered as depicted in Fig. 1.

\section{A. The wave splitting method}

In the time domain, the wave equation can be factorized into a product of two first order differential operators applied to the total pressure field $p$. The solution of the wave equation is then the combination of two independent functions. ${ }^{40}$ Each function is a solution of the corresponding first order differential operator applied to $p$. In the frequency domain, the same idea may be followed. ${ }^{41}$ The Helmholtz equation (16) can be factorized into the following coupled system of first order differential equations:

$$
\left[\frac{1}{\rho_{e}(x, \omega)} \partial_{x} \mp \frac{j \omega}{K_{e}(x, \omega)}\right] p= \pm \text { const } \times \frac{j \omega}{K_{e}(x, \omega)} p^{ \pm},
$$

where $p^{+}=p^{+}(x, \omega)$ and $p^{-}=p^{-}(x, \omega)$ - the sum of which equals $p$-are, respectively, related to well defined forwardpropagating and backward-propagating waves in the case of homogeneous media. It is then useful to introduce the effective sound speed $c_{e}(x, \omega)=\sqrt{K_{e}(x, \omega) / \rho_{e}(x, \omega)}$ and the effective impedance $Z_{e}(x, \omega)=\rho_{e}(x, \omega) c_{e}(x, \omega)$.

The so-called wave splitting transformation, ${ }^{16,27-29}$ together with Eq. (10), takes the form

$$
p^{ \pm}=\frac{1}{2}\left[p \pm Z_{e}(x, \omega) \phi(x) \dot{\mathbf{U}} \cdot \mathbf{n}\right],
$$

wherein $\mathbf{n}$ is the unit normal vector (Fig. 1) and $\phi(x) \dot{\mathbf{U}} \cdot \mathbf{n}$ is the flow of the fluid volume in the $\mathbf{n}$ direction. The use of the effective impedance $Z_{e}$ in Eq. (19) is annoying because the earlier relations must be differentiated with respect to $x$. To avoid this problem, the so-called "vacuum" wave splitting ${ }^{42}$ is preferred. Instead of directly applying the wave splitting transformation to the wave equation in the macroscopically inhomogeneous rigid frame porous medium, it is rather applied to the wave equation in the surrounding homogeneous fluid (the vacuum in electromagnetism). The material prop- erties of the inhomogeneous medium, the effective density $\rho_{e}$, and the effective bulk modulus $K_{e}$ are accounted for in the form of local variations/perturbations of the sound speed $c_{0}$ and of the characteristic impedance $Z_{0}=\rho_{f} c_{0}$ at the location of the slab. Consequently, the vacuum wave splitting transformation is

$$
p^{ \pm}=\frac{1}{2}\left[p \pm Z_{0} \phi(x) \dot{\mathbf{U}} \cdot \mathbf{n}\right] .
$$

A system of linear first order coupled differential equations is obtained by combining Eq. (20) with Eqs. (10) and (11),

$$
\begin{aligned}
& \partial_{x} p^{+}=A^{+}(x, \omega) p^{+}+A^{-}(x, \omega) p^{-}, \\
& \partial_{x} p^{-}=-A^{-}(x, \omega) p^{+}-A^{+}(x, \omega) p^{-},
\end{aligned}
$$

wherein $A^{ \pm}$are $x$-dependent coefficients, accounting for material properties of the inhomogeneous slab

$$
A^{ \pm}=-\frac{j \omega}{2}\left[\frac{Z_{0}}{K_{e}(x, \omega)} \pm \frac{\rho_{e}(x, \omega)}{Z_{0}}\right] .
$$

By definition, the pressure waves $p^{+}(0, \omega), p^{-}(0, \omega)$, and $p^{+}(L, \omega)$ are the incident, reflected, and transmitted pressure waves, respectively denoted as $p^{i}, p^{r}$, and $p^{t}$ in Fig. 1. Then, several physical relations between $p^{+}$and $p^{-}$waves can be defined to solve the system (21). This is done in the following sections.

\section{B. Invariant imbedding and Green's functions}

In the invariant imbedding approach, ${ }^{27-29}$ the problem is imbedded in a family of subproblems. The initial problem is to solve the differential system (21) for the pressure subfields $p^{ \pm}$by performing an integration for all $x$ in $[0, L]$. This problem is decomposed into series of subproblems, consisting in solving the differential system (21) for the $p^{ \pm}$for each sublayer in $[x, L]$. The procedure is initialized with the calculation of $p^{ \pm}$when no layer is present at $x=L$, i.e., in the surrounding homogeneous fluid. Then, the pressure subfields $p^{ \pm}$are re-evaluated following the addition of an infinitely wide layer of thickness $d x$ at $x=L-d x$. This operation is repeated until the interface at $x=0$ is reached. Each $n$th thin layer added at $x=L-n d x$ is homogeneous, with its properties being those of the inhomogeneous material at $L-n d x$. For each addition of a new layer, the continuity of $p$ and of $\phi(x) \dot{\mathbf{U}} \cdot \mathbf{n}$ are implicitly accounted for on both sides of the cumulated slab. Iteratively, the total pressure field inside the inhomogeneous slab is obtained when the last thin layer is added. The problem with this procedure resides in the difficulty of the determination of $p^{ \pm}$at $x=L$, which is mandatory at each iteration.

A way to avoid the determination of this quantity is to introduce the $x$-dependent reflection and transmission coefficients, respectively, denoted by $R(x, \omega)$ and $T(x, \omega)$. They are defined for each subslab between $x$ and $L$ as follows: 


$$
p^{+}\left(L^{+}, \omega\right)=T(x, \omega) p^{+}(x, \omega),
$$

in which $p^{+}(0, \omega), p^{-}(0, \omega)$, and $p^{+}\left(L^{+}, \omega\right)$ are the incident, reflected, and transmitted pressure waves, and consequently $R(0, \omega)=R(\omega)$ and $T(0, \omega)=T(\omega)$ are the usual reflection and transmission coefficients of the total slab. Combining Eqs. (23), (24), and (21), a nonlinear system of first order coupled differential equations is obtained (the frequency dependence is omitted)

$$
\begin{aligned}
& \frac{\partial}{\partial x} R(x)=-A^{-}(x)-2 A^{+}(x) R(x)-A^{-}(x) R^{2}(x), \\
& \frac{\partial}{\partial x} T(x)=-A^{+}(x) T(x)-A^{-}(x) R(x) T(x),
\end{aligned}
$$

wherein $A^{ \pm}$are given by Eq. (22). The resolution of this differential system can be initialized with the values of $R(x, \omega)$ and $T(x, \omega)$ at $x=L$. In the invariant imbedding approach, no subslab is present at $x=L$ and consequently the field at this location is solely the transmitted pressure field, identical to the incident pressure field. Then, $R(L, \omega)=0$ and $T(L, \omega)=1$ at all frequencies. However, Eqs. (26) and (25) are nonlinear. In particular, Eq. (25) takes the form of the Riccati equation.

The reflection and transmission coefficients are strongly linked to interactions inside the material. Krueger and Ochs ${ }^{33}$ introduced the Green's functions approach to linearize time domain scattering problems dealing with the wave splitting method. Green's functions are defined for each subfield $p^{ \pm}$ and depend on the sole material properties. The Green's functions approach ${ }^{20,33}$ allows us to express the internal subfields in terms of the incident wave field $p^{i}=p^{+}(0, \omega)$. Introduced in system (21), this change of variables leads to a linear system of first order differential equations in terms of the Green's functions. However, two boundary conditions are required at two different points: $x=0$ and $x=L$. To obtain an expression of the boundary conditions at the same end point $(x=L)$, "transmission" Green's functions are defined. The latter were first introduced in the time domain as "compact" Green's functions ${ }^{32}$ since they have a compact support in the time variable.

\section{Transmission Green's functions approach}

The internal subfields can be expressed in terms of the transmitted wave field $p^{t}=p^{+}\left(L^{+}, \omega\right)$ instead of the incident wave field. The two transmission Green's functions are then defined as follows: ${ }^{32,42}$

$$
\begin{aligned}
& \forall x \in[0, L], \\
& p^{ \pm}(x, \omega)=G^{ \pm}(x, \omega) p^{+}(L, \omega) .
\end{aligned}
$$

From Eqs. (23), (24), and (27), the relations between Green's functions and the reflection and transmission coefficients turn out to be

\begin{tabular}{|c|c|c|c|c|c|c|}
\hline & $\phi$ & $\tau_{\infty}$ & $\begin{array}{c}\Lambda \\
(\mu \mathrm{m})\end{array}$ & $\begin{array}{c}\Lambda^{\prime} \\
(\mu \mathrm{m})\end{array}$ & $\begin{array}{c}R_{f} \\
\left(\mathrm{~N} \mathrm{~s} \mathrm{~m}^{-4}\right) \\
\end{array}$ & $\begin{array}{c}\text { Thickness } \\
(\mathrm{mm})\end{array}$ \\
\hline Layer 1 & 0.96 & 1.07 & 273 & 672 & 2843 & 7.1 \\
\hline Layer 2 & 0.99 & 1.001 & 230 & 250 & 12000 & 10.0 \\
\hline
\end{tabular}

$$
\begin{aligned}
& R(x, \omega)=G^{-}(x, \omega) / G^{+}(x, \omega), \\
& T(x, \omega)=T(L, \omega) / G^{+}(x, \omega) .
\end{aligned}
$$

TABLE I. Properties of the two-layer medium studied.

Since the boundary conditions are known for $R$ and $T$ at $x=L[R(L, \omega)=0$ and $T(L, \omega)=1]$, the boundary conditions for $G^{ \pm}$are

$$
\begin{aligned}
& G^{+}(L, \omega)=1 . \\
& G^{-}(L, \omega)=0 .
\end{aligned}
$$

The introduction of Eq. (27) into Eq. (21) leads to a linear system of first order differential equations in terms of the transmission Green's functions with the two boundary conditions at the single end point $x=L$,

$$
\begin{aligned}
& \partial_{x} G^{+}=A^{+}(x \omega) G^{+}+A^{-}(x, \omega) G^{-}, \\
& \partial_{x} G^{-}=-A^{-}(x, \omega) G^{+}-A^{+}(x, \omega) G^{-},
\end{aligned}
$$

wherein $G^{ \pm}=G^{ \pm}(x, \omega)$ and the functions $A^{ \pm}(x, \omega)$ are given by Eq. (22).

System (31) is solved by integrating in the $-x$ direction from $x=L$ to $x=0$ following the procedure exposed in Sec. III B, together with an explicit fourth-order Runge-Kutta method.

Once system (31) is solved for $G^{+}$and $G^{-}$, the internal pressure field, and also the reflected and transmitted fields, can be determined through the change of variables in Eqs. (27) and (20).

\section{SIMULATIONS ON A MULTILAYERED POROUS MEDIUM}

In order to validate the WS-TGF method, calculations are performed for a known two-layered porous medium configuration considered as a single rigid frame porous slab. Each layer of the slab is a highly porous polyurethane foam saturated by air. The characteristic properties of each layer have been determined by ultrasonic methods ${ }^{18}$ and are given in Table I.

The choice of this configuration is motivated by the fact that the results of the WS-TGF method can be compared with known results from the classical TMM, ${ }^{15}$ and with experimental measurement.

To consider the slab as a single inhomogeneous material, the jump discontinuities in the two-layered system are smoothed by using the following analytical continuous and continuously differentiable function:

$$
I(x)=1+\frac{C}{2}\left[1+\operatorname{erf}\left(\frac{x-x_{0}}{r}\right)\right],
$$

wherein $C$ is the step value, which is different for each parameter in Table $\mathrm{I}$, erf is the error function, $x_{0}$ is the position of the jump, and $r$ corresponds to the steepness of the continuous jump such that the smaller is $r$, the steeper is the jump. 

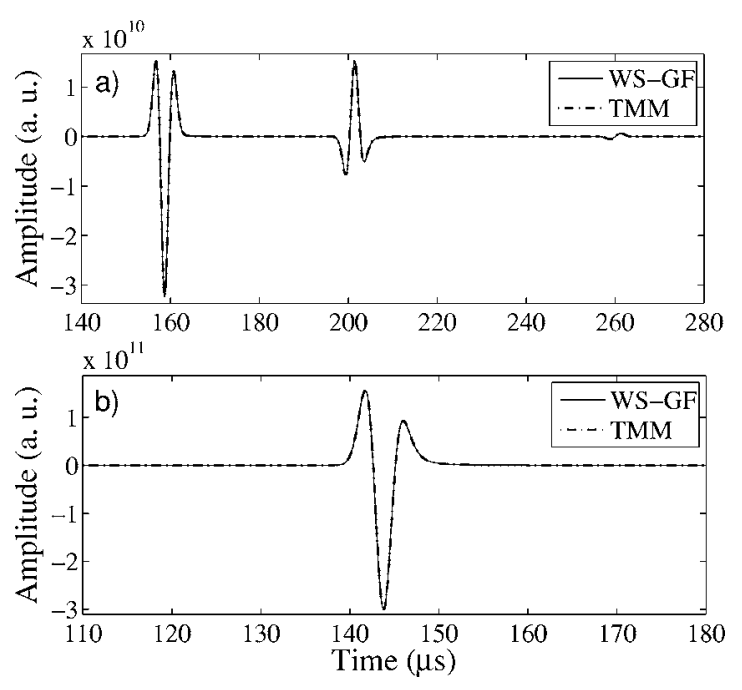

FIG. 2. Reflected (panel a) and transmitted (panel b) fields calculated by the TMM method (solid curve) and by the WS-TGF method (dashed curve) for a two-layer porous material solicited by an incident plane wave. The angle of incidence is $\theta=0^{\circ}$.

\section{A. Simulations at normal incidence}

The incident plane wave takes the form of a Ricker wavelet, e.g., a second order time derivative of a Gaussian distribution, whose spectrum is

$$
S(\omega)=\frac{-\left(\pi \nu_{0}\right)^{2} \omega^{2}}{2 \sqrt{\pi}\left(\pi \nu_{0}\right)^{3}} \exp \left(-j \frac{\omega}{\nu_{0}}-\frac{\omega^{2}}{\left(2 \pi \nu_{0}\right)^{2}}\right) .
$$

This signal is similar to those provided by capacitive transducers. The central frequency $\nu_{0}$ of the incident signal is $200 \mathrm{kHz}$.

In order to study the influence of the steepness $r$ of the smoothed jump on the amplitude of the reflected and transmitted fields at the interface located at $x=x_{0}$, the response of the slab is simulated for several values of $r$. To obtain a very accurate fit between the TMM and the WS-TGF methods, it is found that this parameter must satisfy the empirical relation $r<0.5 \times d x$, wherein $d x$ is the discretization step. For a steepness parameter bigger than $0.5 d x$, a small difference between the signals simulated by both methods may be noticed, and thus later between the WS-TGF method and experiments.

Remark: This choice of steepness parameter could lead numerically to a Heaviside distribution. This was found to not be a numerical problem, but if such a problem arises, a logarithmic discretization step could be used over a small interval around $x_{0}$, as was done in Ref. 31.

Figure 2 compares the reflected and transmitted signals calculated by the WS-TGF method with those calculated by the TMM method for a normally incident pressure field. The two curves cannot be distinguished.

\section{B. Simulations at oblique incidence}

At oblique incidence, the numerical procedure of the WS-TGF method must be modified. Let us introduce the wave number $k_{e}(x, \omega)=\omega / c_{e}(x, \omega)$ in the inhomogeneous slab and the wave number $k_{0}=\omega / c_{0}$ in the surrounding fluid, i.e., in the air. At oblique incidence, the wave vectors are projected on the $x$ and $y$ axis ${ }^{43}$ so that $k_{j}=k_{j}^{x} \mathbf{i}_{x}+k_{j}^{y} \mathbf{i}_{y}$, with $j$ $=0$ or $e$, and $\mathbf{i}_{x}$ and $\mathbf{i}_{y}$ as the unit vectors along the positive $x$ and $y$ axis, respectively. Then, $k_{j}=\sqrt{\left(k_{j}^{x}\right)^{2}+\left(k_{j}^{y}\right)^{2}}$, with $\mathfrak{R}\left(k_{j}^{y}\right)$ $\geq 0$ and $\Im\left(k_{j}^{y}\right) \leq 0$ to satisfy the outgoing wave condition. It follows from the application of the boundary conditions that $k_{j}^{y}$ is constant and equal to $k_{0} \sin \theta$. Separation of variables implies that

$$
\begin{aligned}
& p(x, y, \omega)=\bar{p}(x, \omega) e^{-j k_{0} y \sin \theta}, \\
& \phi(x) \dot{\mathbf{U}}(x, y, \omega)=\phi(x) \overline{\dot{\mathbf{U}}}(x, \omega) e^{-j k_{0} y \sin \theta}
\end{aligned}
$$

in the inhomogeneous layer. The equations of motion (10) and (11) take the form

$$
\begin{aligned}
& -\frac{\partial \bar{p}}{\partial x}=j \omega \rho_{e} \phi(x) \overline{\dot{\mathbf{U}}}(x, \omega) \cdot \mathbf{i}_{x}, \\
& -\frac{\sin \theta}{c_{0}} \bar{p}=j \omega \rho_{e} \phi(x) \overline{\dot{\mathbf{U}}}(x, \omega) \cdot \mathbf{i}_{y}, \\
& j \omega \bar{p}=-K_{e}\left\{\frac{\partial \phi(x) \overline{\dot{\mathbf{U}}}(x, \omega) \cdot \mathbf{i}_{x}}{\partial x}\right. \\
& \left.-j \omega \frac{\sin \theta}{c_{0}}\left[\phi(x) \overline{\dot{\mathbf{U}}}(x, \omega) \cdot \mathbf{i}_{y}\right]\right\} .
\end{aligned}
$$

By introducing Eq. (37) into Eq. (38) in order to get rid of $\phi(x) \overline{\dot{\mathbf{U}}}(x, \omega) \cdot \mathbf{i}_{y}$, the equations of motion reduce to

$$
\begin{aligned}
& \frac{\partial \phi(x) \overline{\dot{\mathbf{U}}}(x, \omega) \cdot \mathbf{i}_{x}}{\partial x}=-j\left(\frac{\omega}{K_{e}}-\frac{\omega \sin ^{2} \theta}{c_{0}^{2} \rho_{e}}\right) \bar{p}, \\
& \frac{\partial \bar{p}}{\partial x}=-j \omega \rho_{e} \phi(x) \overline{\dot{\mathbf{U}}}(x, \omega) \cdot \mathbf{i}_{x} .
\end{aligned}
$$

An angle-dependent effective sound speed $\bar{c}_{e}(x, \omega, \theta)$ and an effective characteristic impedance $\bar{Z}_{e}(x, \omega, \theta)$ are defined as follows:

$$
\begin{aligned}
& \bar{c}_{e}(x, \omega, \theta)=c_{e}(x, \omega)\left[1-\frac{c_{e}^{2}(x, \omega)}{c_{0}^{2}} \sin ^{2} \theta\right]^{-1 / 2}, \\
& \bar{Z}_{e}(x, \omega, \theta)=Z_{e}(x, \omega)\left[1-\frac{c_{e}^{2}(x, \omega)}{c_{0}^{2}} \sin ^{2} \theta\right]^{-1 / 2} .
\end{aligned}
$$

Thus, the effective sound speed $c_{e}$ and characteristic impedance $Z_{e}$ in equations for normal incidence just have to be replaced by the angle-dependent ones, and the procedure exposed in Sec. III can be used to solve the problem when the slab is solicited by an obliquely incident plane wave. The effective bulk modulus $K_{e}(x, \omega)$ must also be replaced by $\bar{K}_{e}(x, \omega, \theta)=K_{e}(x, \omega)\left\{1-\left[c_{e}^{2}(x, \omega) / c_{0}^{2}\right] \sin ^{2} \theta\right\}^{-1}$ in Eq. (22). Figure 3 compares the reflected and transmitted fields calculated by the WS-TGF method with those of the TMM method, when the two-layered system is solicited by a plane wave at incident angle $\theta=40^{\circ}$. The two curves cannot be 6 

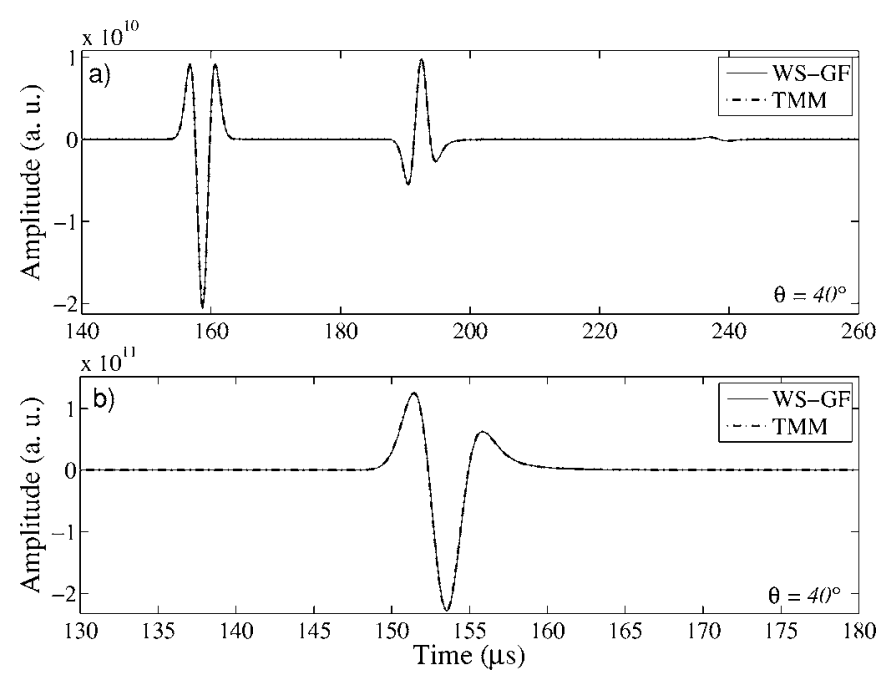

FIG. 3. Reflected (panel a) and transmitted (panel b) fields calculated by the TMM method (solid curve) and by the WS-TGF method (dashed curve) for a two-layer porous material solicited by an incident plane wave. The angle of incidence is $\theta=40^{\circ}$.

\section{Frequency domain simulations}

Figure 4 compares the reflection and transmission coefficients calculated by the WS-TGF method with those of the TMM for angles of incidence $\theta$ in $[0, \pi / 2]$ and angular frequencies $\omega$ in $[150 \mathrm{kHz}, 250 \mathrm{kHz}]$. The curves again match very well.

Figure 4 calls for several remarks. The transmission coefficient decreases as the incidence angle increases. This is due to the fact that at oblique incidence the distance over which the wave propagates in the slab is longer. The reflection coefficient admits a minimum around $55^{\circ}(0.9 \mathrm{rad})$. For
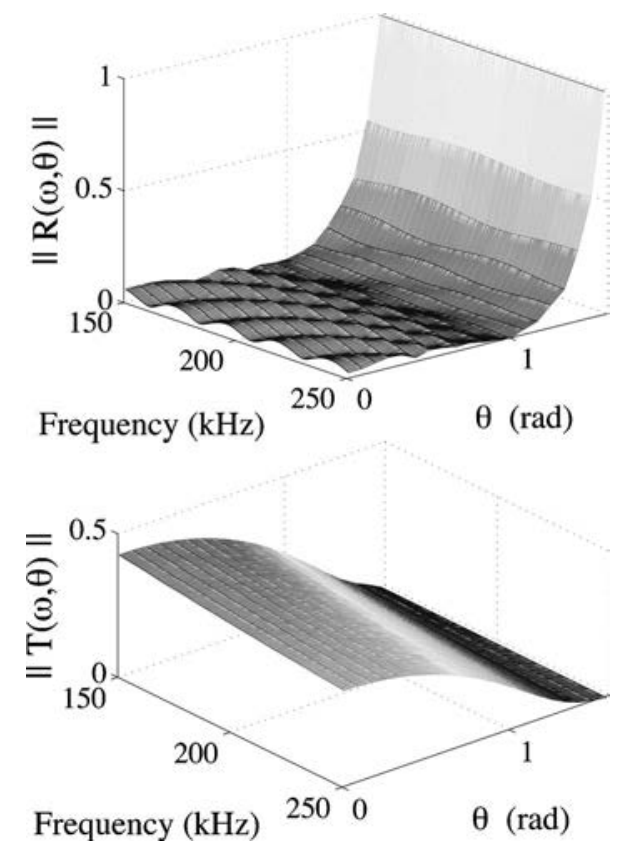

FIG. 4. Modulus of the reflection coefficient $R(\omega, \theta)$ (upper panel) and the transmission coefficient $T(\omega, \theta)$ (lower panel) for several angles of incidence $\theta$, calculated by the WS-TGF method and by the TMM method, for a two-layer porous material. The two curves in each panel cannot be distinguished.

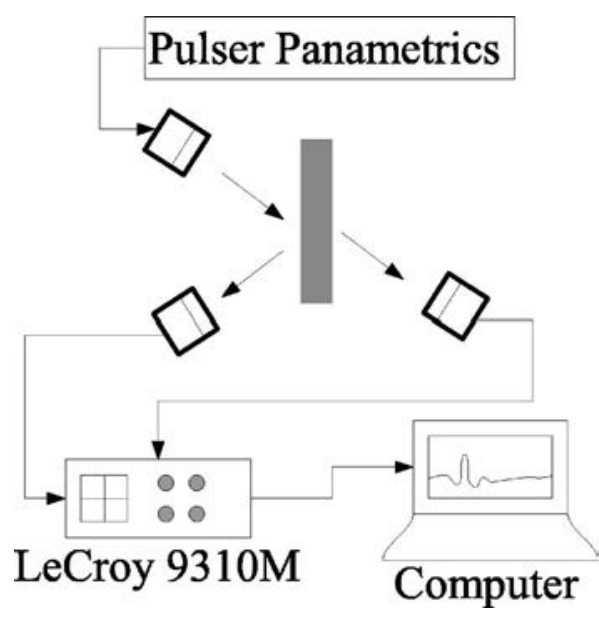

FIG. 5. Experimental setup. The transducers are Ultran air-coupled piezoelectric transducers.

any angle of incidence $\theta$ in $[0, \pi / 2]$, transmitted pressure waves exist [total reflection occurs only for an angle of incidence $\left.90^{\circ}(\pi / 2 \mathrm{rad})\right]$, which is another way of saying that no critical angle exists for this configuration.

The accuracy of the macroscopically inhomogeneous rigid frame porous materials model and the WS-TGF method was demonstrated by means of numerical examples. This validation is now completed with the help of experimental results.

\section{EXPERIMENTAL RESULTS}

The principle of the experiment is shown in Fig. 5. An airborne ultrasonic wave is generated and detected at normal or oblique incidence by air-coupled Ultran piezoelectric transducers in a frequency range between 150 and $250 \mathrm{kHz}$.

At normal incidence, the reflection experiments are performed in the pulse-echo mode ${ }^{44}$ with only one transducer. The experimental and calculated reflected and transmitted fields in Fig. 6 are in good agreement, as are the reflection and transmission coefficients in Fig. 7. No regularization method was used to optimize the calculation of the reflection and transmission coefficients. Therefore, the energy of the

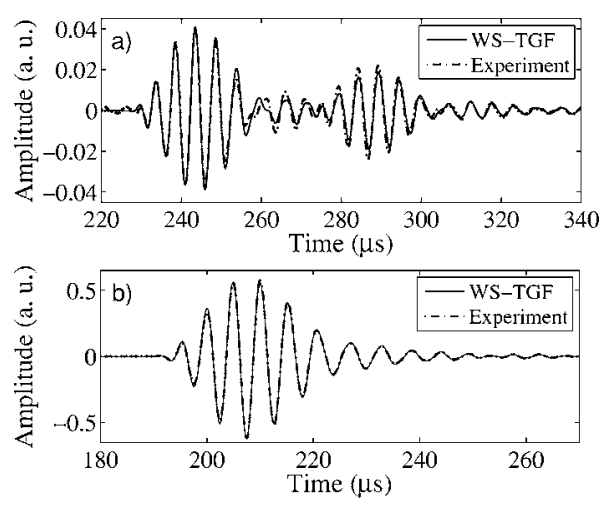

FIG. 6. Comparison of the reflected (a) and transmitted (b) fields obtained by experiments (dashed curve) with those obtained by the WS-TGF method 7 


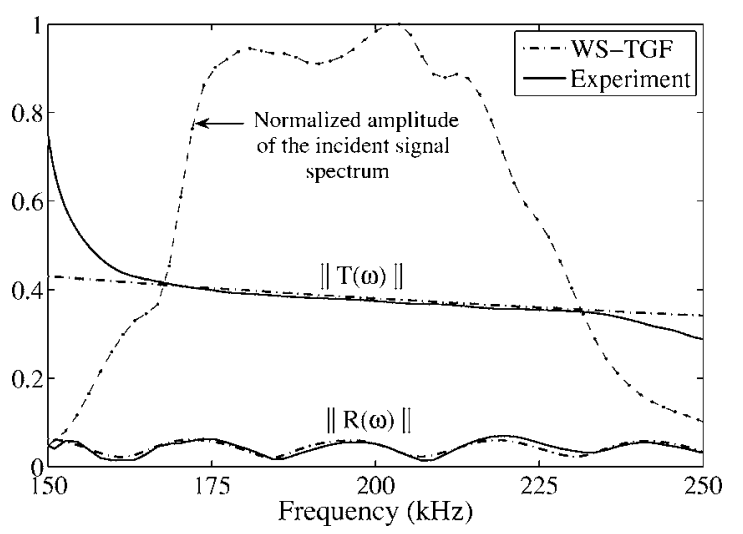

FIG. 7. Comparison of the reflection and transmission coefficients obtained by experiments (dashed curve) with those obtained by the WS-TGF method (solid curve). The angle of incidence is $\theta=0^{\circ}$.

incident signal being too small outside the frequency bandwidth, our experimental reflected and transmitted coefficients are accurate only in this bandwidth.

It is more difficult to carry out the experiments at oblique incidence, because: (i) the signal-to-noise ratio decreases with increasing incident angle and (ii) the two foams are not glued together, which may induce a possible thin air layer between the two porous layers whose influence is greater at oblique incidence. Averaging over several synchronized captures on the oscilloscope leads to an important reduction of noise and thus to a more efficient extraction of the reflected signal. Comparisons between experimental and simulated reflected and transmitted signals are presented in Figs. 8 and 9.

Figure 10 depicts the simulated and experimental reflection and transmission coefficients for an incident angle of $30^{\circ}$. In this figure, the incident signal spectrum is different from the one in Fig. 7 because the experimental setups used to get both reference signals are different (use of a single transducer in pulse echo mode at normal incidence and of two transducers at oblique incidence to measure signals reflected by a flat steel target).

The numerical results computed by the WS-TGF method are in very good agreement with the corresponding experi-

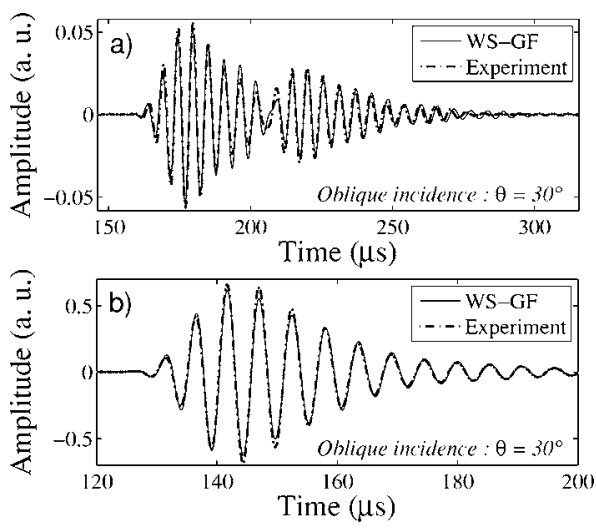

FIG. 8. Comparison of the reflected (a) and transmitted (b) fields obtained by experiment (dashed curve) with those computed by the WS-TGF method (solid curve). The angle of incidence is $\theta=30^{\circ}$.
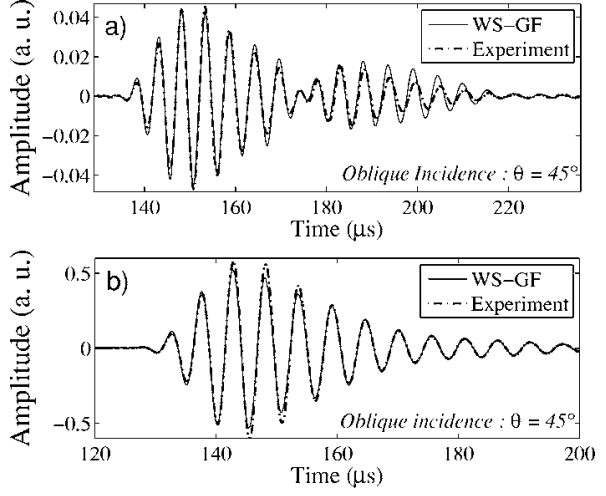

FIG. 9. Comparison of the reflected (a) and transmitted (b) fields obtained by experiment (dashed curve) with those computed by the WS-TGF method (solid curve). The angle of incidence is $\theta=45^{\circ}$.

mental results. This validates both the method of resolution and the system of equations for an inhomogeneous rigid frame porous medium.

\section{ASSUMPTIONS ON INTERNAL PRESSURE FIELD AND OTHER PROPERTY PROFILES}

Macroscopically inhomogeneous porous materials offer the possibility of wider applications in sound absorbing material design than their macroscopically homogeneous counterparts. In the following, we present some examples of absorbing material design.

First, the acoustical properties of the previously-studied two-layered slab (see Table I) are considered. A porous slab of thickness $L$ with linearly varying parameters is chosen such that its properties match those of the first layer on one side $(x=0)$ and those of the second layer on the other side ( $x=L)$. This material could be constructed, for example, by compressing a foam of properties matching those of the first layer, denoted by $\mathcal{F}_{1}$, or by expanding a foam whose properties match those of the second layer, denoted by $\mathcal{F}_{2}$. Indeed, for a considered mesoscopic constant volume, compression results in a decrease of the pore volume. This leads to a decreasing porosity and characteristic lengths. The variations of the tortuosity and flow resistivity as functions of the deformation applied to the medium are dependent on the

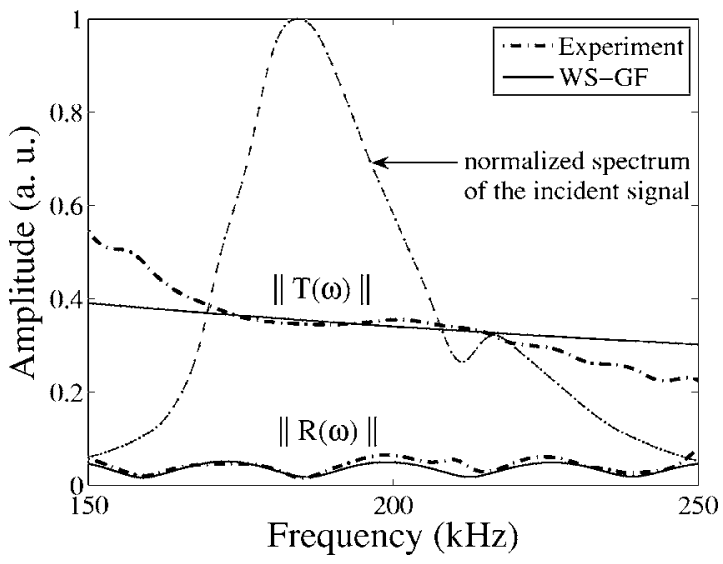

FIG. 10. Comparison of the reflection and transmission coefficients obtained by experiment (dashed curve) with those computed by the WS-TGF 

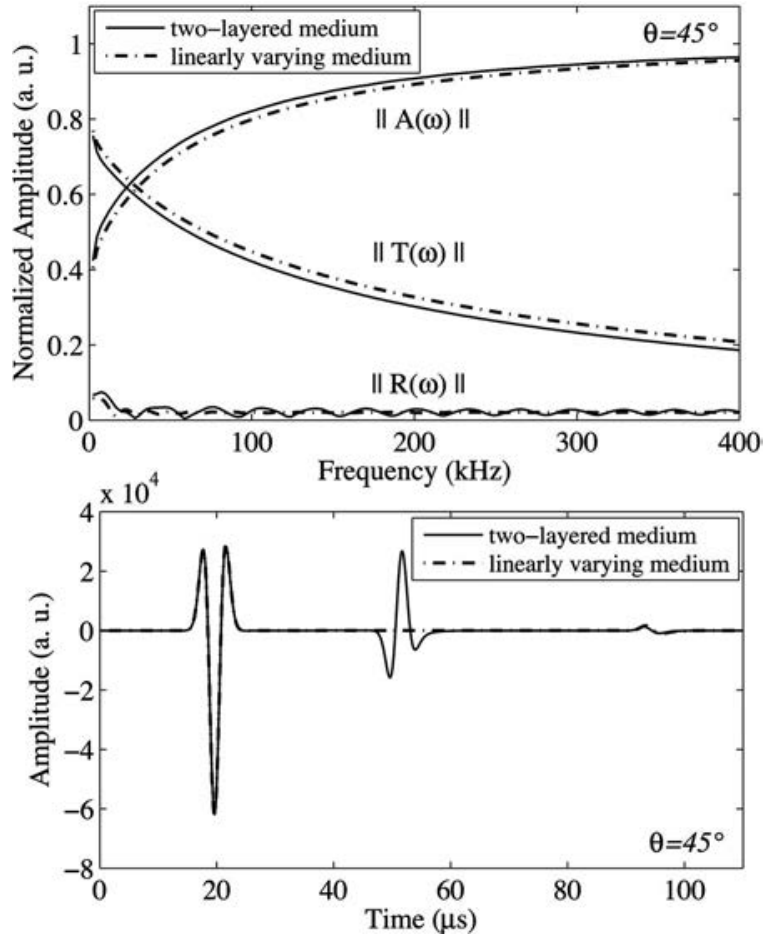

FIG. 11. Upper panel: comparison of the reflection, transmission and absorption coefficients. Lower panel: comparison of the reflected pressure fields between the two-layered medium (solid curve) and the linearly varying properties medium (dashed curve). The angle of incidence is $\theta=45^{\circ}$.

pore structure and so can be different from one porous medium to the other. Here we assume that both quantities increase. The gradient of properties inside the slab is chosen to be constant so that $\phi(x), \tau_{\infty}(x), \Lambda(x), \Lambda^{\prime}(x)$, and $R_{f}(x)$ vary linearly with $x$.

The acoustical performance of a sample with linearly varying properties can be evaluated by use of our set of equations and method of resolution, i.e., the WS-TGF method. According to the properties values in Table I and to measurements realized on each layer, $\mathcal{F}_{2}$, i.e., the melamine foam, is more absorbing than $\mathcal{F}_{1}$ (the flow resistivity is a good indicator of the absorption of a medium). Consequently, the materials with linearly varying properties imply an increase (respectively, decrease) of the absorption and reflection coefficients and a decrease (respectively, increase) of the transmission coefficient compared to the ones of foam $\mathcal{F}_{1}$ (respectively, $\mathcal{F}_{2}$ ).

A comparison is made in the frequency domain between the material with linearly varying parameters and the corresponding two-layered foam slab concerning the reflection and transmission coefficients $R(\omega)$ and $T(\omega)$ (i.e., the ratios of the amplitudes of the reflected and transmitted signals over the amplitude of the incident signal) and the absorption coefficient $A(\omega)$, defined as $\|A(\omega)\|=1-\|R(\omega)\|^{2}-\|T(\omega)\|^{2}$. This is depicted in Fig. 11 (upper panel) for a $45^{\circ}$ incidence angle. For both profiles, the value of each coefficient is of the same order. The two-layered medium is nevertheless less transmitting and more absorbing. This is mainly due to the fact that the second layer is the more absorbing of the two in the two-layered slab.

A comparison of the reflected pressure fields in the time
TABLE II. Properties of a two-layer medium simulated with strong variations of its properties.

\begin{tabular}{|c|c|c|c|c|c|c|}
\hline & $\phi$ & $\tau_{\infty}$ & $\begin{array}{c}\Lambda \\
(\mu \mathrm{m})\end{array}$ & $\begin{array}{c}\Lambda^{\prime} \\
(\mu \mathrm{m})\end{array}$ & $\begin{array}{c}R_{f} \\
\left(\mathrm{~N} \mathrm{~s} \mathrm{~m}{ }^{-4}\right)\end{array}$ & $\begin{array}{l}\text { thick } \\
(\mathrm{mm})\end{array}$ \\
\hline Layer 1 & 0.6 & 2.5 & 130 & 320 & 30000 & 7 \\
\hline Layer 2 & 0.96 & 1.07 & 273 & 672 & 2843 & 10 \\
\hline
\end{tabular}

domain between the two-layered material and the foam with linearly varying properties is depicted in Fig. 11 (lower panel). A particular feature of the linearly varying foam is that the reflected field is composed of two pulses, those reflected on both sides of the slab, while it is composed of three pulses in the case of the two-layered foam: the two reflected ones on both sides of the slab and a third one reflected on the interface between the two layers.

The internal pressure field [mapped with the help of Eq. (34)] and the internal energy might both be useful to understand the behavior of inhomogeneous materials. However, for the set of material properties previously used, whose values are close, the change in the direction of propagation is too small to be noticed in a snapshot of the internal pressure. In Table II we give the acoustical properties of a more suitable configuration in which a layer is replaced by a foam with smaller pore geometry and more complex structure, which induces a lower porosity, higher tortuosity and smaller characteristic lengths. These values imply an important impedance mismatch at the interface between the two layers.

Snapshots of the internal pressure fields of both the twolayered and linearly varying properties slab, as well as a comparison between the reflection and transmission coefficients, are given in Fig. 12. In both snapshots of the internal pressure field, the orientation of the wave fronts are the same at $x=0$ and $x=L$ as expected. The amplitudes are also of the same order. The ray direction, which is perpendicular to the curved wave fronts in the snapshots, is approaching the $y$ direction (grazing direction). The curvature of the wave front implies that less energy is transmitted through the porous slab as shown in Fig. 12 in comparison with Fig. 11. In the case of a two-layered medium, the impedance mismatch at the internal interface at $x=7 \mathrm{~mm}$ gives rise to interferences in the first layer, which are easily visible in the snapshots of the internal pressure field in Fig. 12, and to strong oscillations of the reflection and transmission coefficients. The interferences are caused by the inner strong reflections. No such oscillations and interferences are noticed in the case of the linearly varying medium, which is another way of saying that no important impedance mismatch exists within the slab. This interference pattern could be used in the detection and localization of important impedance mismatches in porous materials along the direction of propagation, like it is the case of interfaces for the present unidimensional model, or of more complicated objects like cracks, holes, porous or solid inclusions for a two- or three-dimensional model.

If we solicit the medium with linearly varying properties from the other side, the ray path will have a tendency of becoming parallel to the $x$ direction at $x=0$. It might be pos- 

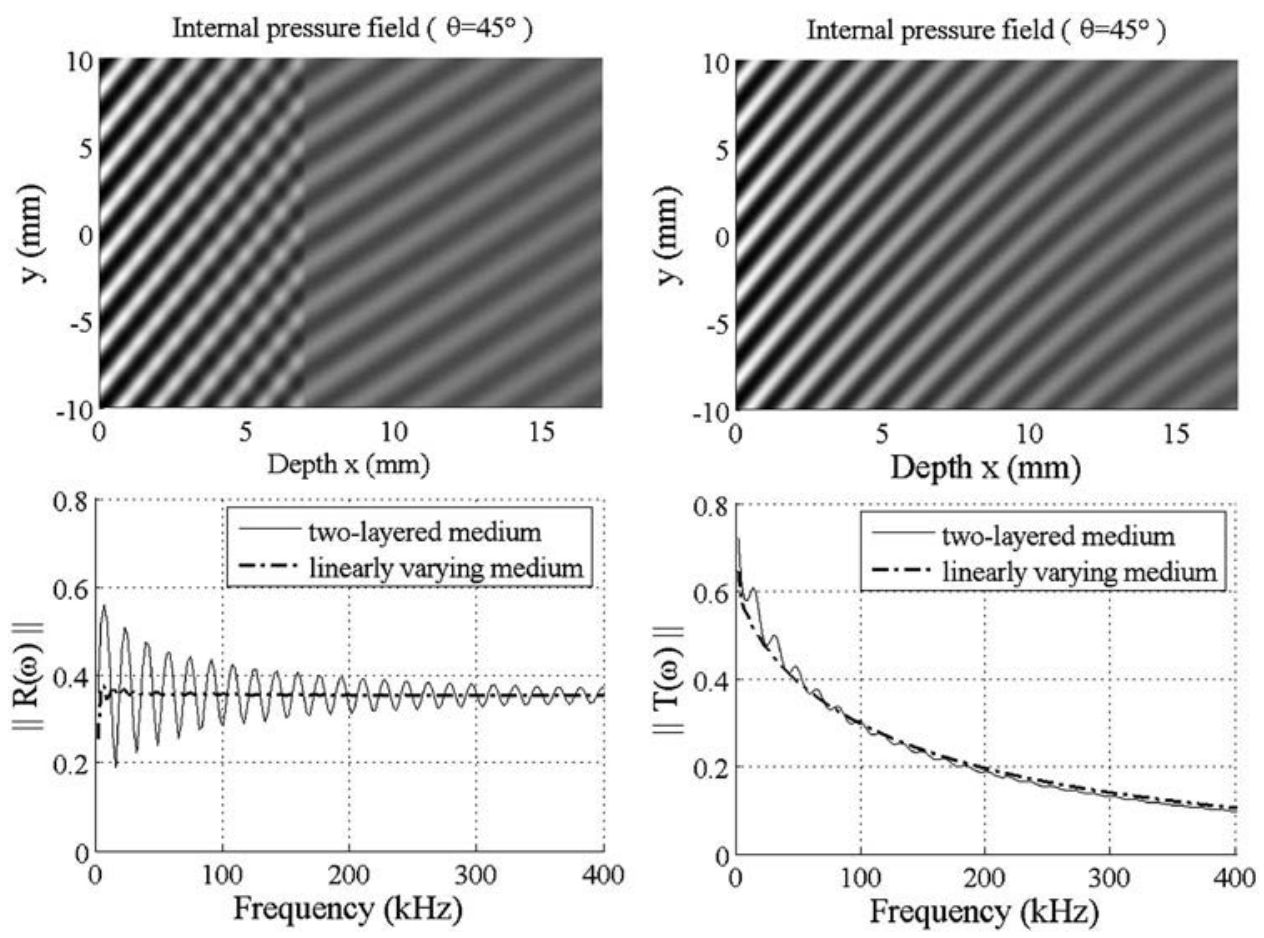

FIG. 12. Top panels: snapshots of the internal pressure fields for the two-layered medium of Table II and for the corresponding linearly varying properties medium with constant gradient of properties. Bottom panels: comparison between the reflection and transmission coefficients, $\|R(\omega)\|$ and $\|T(\omega)\|$. Oblique incidence $\theta=45^{\circ}$.

with first a decrease in sound speed to limit reflection, and then an increase to limit transmission. Parabolic profiles, connecting the properties of foam 2 in Table II to those of foam 1 , and back to those of foam 2 , have been tried, in order to reduce both the reflection and the transmission from the whole slab. The results are shown in Fig. 13. Comparison with Fig. 12 shows that the reflected energy is reduced, that
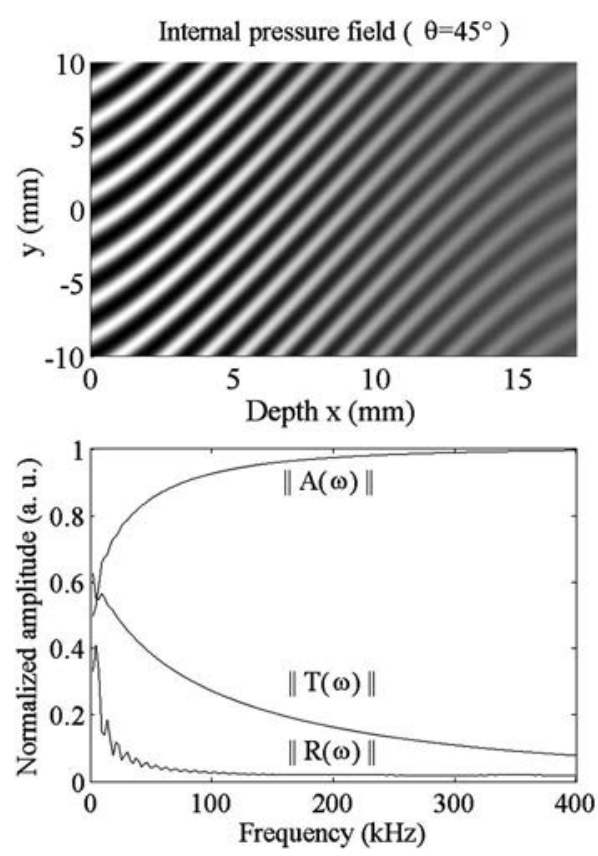

FIG. 13. Snapshot of the internal pressure field (top panel), and the reflection $\|R(\omega)\|$, transmission $\|T(\omega)\|$, and absorption $\|A(\omega)\|$ coefficients (bottom panel), for a parabolic profile of properties. The angle of incidence is $\theta$ $=45^{\circ}$. the values of both transmission coefficients are of the same order and that the considered medium with parabolic properties profiles is more absorbing than the related material with linearly varying properties.

The previous examples indicate the wide range of applications that can be investigated with the help of the wave equation and the resolution method described in this article. Nevertheless, the behavior of simulated materials using homogeneous plane waves is perhaps not realistic, especially to visualize the acoustical ray path and to localize variations of impedance with accuracy. The solicitation by a line source might be more suitable and is known to enable the excitation of evanescent waves inside the slab. ${ }^{45}$ The variation of material properties of a compressed foam should also be studied.

\section{CONCLUSION}

A model of the acoustic response of macroscopically inhomogeneous elastic frame porous materials was derived from the alternative Biot's theory of 1962. This formulation is the only one which is valid for this type of heterogeneity, as previously stated in. ${ }^{2,34,35}$ As a first verification, a study was made of wave propagation in rigid frame inhomogeneous porous materials for which the inhomogeneous equivalent fluid model was obtained. A fast and stable numerical method, deriving from the wave splittingtransmission Green's function approach, was developed to solve the inhomogeneous Helmholtz Eq. (16). A validation of this method was made on the example of a two-layered medium, by comparison to the exact solution (obtained by the transfer matrix method) ${ }^{15}$ and to experimental results, for both normal and oblique plane wave incidence. In the nu- 
merical procedure, the jump of properties between the layers was accounted for in the form of a single continuous function. Both internal and external pressure fields were determined as well as the reflection and transmission coefficients. The internal fields and reflection/absorption/transmission coefficients were computed in the case of materials with a gradient of properties. These examples illustrated the possibility of designing acoustically absorbing materials by controlling the gradient of parameters. This last point requires further investigation, in particular, how the properties vary when the foam is compressed. Another possible application of the equations and method of resolution derived here is the development of an optimized inversion procedure to characterize macroscopically inhomogeneous porous materials.

${ }^{1}$ M. A. Biot, J. Acoust. Soc. Am. 28, 168 (1956); M. A. Biot, J. Acoust. Soc. Am. 28, 179 (1956).

${ }^{2}$ M. A. Biot, J. Appl. Phys. 33, 1482 (1962).

${ }^{3}$ N. Sebaa, Z. E. A. Fellah, M. Fellah, E. Ogam, A. Wirgin, F. G. Mitri, C. Depollier, and W. Lauriks, J. Acoust. Soc. Am. 120, 1816 (2006).

${ }^{4}$ C. Boutin, P. Royer, and J.-L. Auriault, Int. J. Solids Struct. 35, 4709 (1998).

${ }^{5}$ O. Matsuda and O. B. Wright, J. Opt. Soc. Am. B 19, 3028 (2002).

${ }^{6}$ J. Lundstedt and M. Norgren, Prog. Electromagn. Res. 43, 1 (2003).

${ }^{7}$ L. M. Brekhovskikh and Y. P. Lysanov, Fundamentals of Ocean Acoustics (Springer, Berlin, 1991).

${ }^{8}$ J. L. Buchanan, R. P. Gilbert, A. Wirgin, and Y. S. Xu, Marine Acoustics: Direct and Inverse Problems (SIAM, Philadelphia, 2004).

${ }^{9}$ A. J. Robins, J. Acoust. Soc. Am. 103, 1337 (1998).

${ }^{10}$ R. H. Stolt and B. Jacobs, Stanford Exploration Project Report 24, 135 (1980).

${ }^{11}$ J. G. Berryman and R. R. Greene, Geophys. J. 45, 213 (1980).

${ }^{12}$ S. Torquato, Random Heterogeneous Materials: Microstructure and Macroscopic Properties (Springer, New York, 2002).

${ }^{13}$ D. L. Johnson, J. Koplik, and R. Dashen, J. Fluid Mech. 176, 379 (1987).

${ }^{14}$ K. Attenborough, Phys. Rep. 82, 179 (1982).

${ }^{15}$ J. F. Allard, Propagation of Sound in Porous Media: Modeling Sound Absorbing Materials (Chapman and Hall, London, 1993).

${ }^{16}$ L. De Ryck, J. P. Groby, P. Leclaire, A. Wirgin, W. Lauriks, C. Depollier, and Z. E. A. Fellah, Appl. Phys. Lett. 90, 181901 (2007).

${ }^{17}$ Y. Champoux and J. F. Allard, J. Appl. Phys. 70, 1975 (1991).

${ }^{18}$ P. Leclaire, W. Kelders, L. Lauriks, N. R. Brown, M. Melon, and B. Castagnède, J. Appl. Phys. 80, 2009 (1996).
${ }^{19}$ V. Tournat, V. Pagneux, D. Lafarge, and L. Jaouen, Phys. Rev. E 70, 026609 (2004).

${ }^{20}$ G. Kristensson, A. Karlsson, and S. Rikte, in Scattering, edited by R. Pike and P. Sabatier (Academic, San Diego, 2002), pp. 277-294.

${ }^{21}$ L. Knopoff, Bull. Seismol. Soc. Am. 54, 431 (1964).

${ }^{22}$ R. Hähner, in Scattering, edited by R. Pike and P. Sabatier (Academic, San Diego, 2002), pp. 75-94.

${ }^{23}$ P. Sabatier, in Scattering, edited by R. Pike and P. Sabatier (Academic, San Diego, 2002), pp. 717-725.

${ }^{24}$ R. G. Newton, in Scattering, edited by R. Pike and P. Sabatier (Academic, San Diego, 2002), pp. 686-701.

${ }^{25}$ J.-P. Groby and C. Tsogka, in Mathematical and Numerical Aspects of Wave Propagation WAVES 2003, edited by G. C. Cohen and E. Heikkola (Springer, Berlin, 2003), pp. 911-915.

${ }^{26}$ Z. Alterman, J. Phys. Earth 16, 113 (1968).

${ }^{27}$ G. Kristensson and R. J. Krueger, J. Math. Phys. 27,1667 (1986); G. Kristensson and R. J. Krueger, J. Math. Phys. 27, 1683 (1986).

${ }^{28}$ G. Kristensson and R. J. Krueger, J. Math. Phys. 28, 360 (1987).

${ }^{29}$ G. Kristensson and R. J. Krueger, Inverse Probl. 5, 375 (1989).

${ }^{30}$ P. Sabatier, J. Math. Phys. 41, 4082 (2000).

${ }^{31}$ J. P. Groby, L. De Ryck, P. Leclaire, A. Wirgin, W. Lauriks, R. P. Gilbert, and Y. S. Xu, Math. Methods Appl. Sci. 30, 91 (2007).

${ }^{32}$ S. He, J. Math. Phys. 34, 4628 (1993).

${ }^{33}$ R. J. Krueger and L. Ochs, Wave Motion 11, 525 (1989).

${ }^{34}$ S. R. Pride and J. G. Berryman, J. Mech. Phys. Solids 46, 719 (1998).

${ }^{35}$ J. M. Carcione, Wavefield in Real Media: Wave Propagation in Anisotropic, Anelastic and Porous Media (Pergamon, New York, 2001).

${ }^{36}$ M. A. Biot and D. G. Willis, J. Appl. Mech. 36, 594 (1957).

${ }^{37}$ H. Deresiewicz and R. Skalak, Bull. Seismol. Soc. Am. 53, 783 (1963).

${ }^{38}$ T. Bourbié, O. Coussy, and B. Zinszner, Acoustics of Porous Media (Technip, Paris, 1987).

${ }^{39}$ M. Fellah, Z. E. A. Fellah, and C. Depollier, Signal Process. 86, 2658 (2006).

${ }^{40} \mathrm{~d}$ 'Alembert, Opuscules Mathémathiques ou Mémoires sur Différens Sujets de Géométrie, de Méchanique, d'Optique, d'Astronomie (C.-A. Jombert, Paris, 1761-1780).

${ }^{41}$ B. A. Auld, Acoustic Fields and Waves in Solids (Krieger, Malabar, FL, 1990).

${ }^{42}$ O. Forslund and S. He, Prog. Electromagn. Res. 19, 147 (1998).

${ }^{43}$ C. Rorres, J. Bai, P. C. Pederson, and O. J. Tretiak, J. Acoust. Soc. Am. 78, 1851 (1985).

${ }^{44}$ Z. E. A. Fellah, S. Berger, W. Lauriks, C. Depollier, P. Trompette, and J. Y. Chapelon, J. Appl. Phys. 93, 9352 (2003).

${ }^{45}$ J. P. Groby et al., Symposium on the Acoustics of Poro-Elastic Materials, (ENTPE, Lyon, France, 2005), pp. 53-60. 